\title{
Impacto del modelo dual de TSU en logística área cadena de suministros en la ciudad de San Luis Potosí
}

\section{Impact of TSU dual model on logistics supply chain area in the city of San Luis Potosí}

\author{
CHAVÉZ-GOVEA, Roberto*†, CASTRO-LÓPEZ José David, SALAZAR-SALAS Elizabeth y \\ CEDILLO-CAMPOS, Hermes Orestes
}

Universidad Tecnológica de San Luis Potosí

ID $1^{\text {er }}$ Autor: Roberto, Chávez-Govea / ORC ID: 0000-0001-5538-0774

ID $1^{\text {er }}$ Coautor: José David, Castro-López / ORC ID: 0000-0003-4976-213X

ID $2^{\text {do }}$ Coautor: Elizabeth, Salazar-Salas / ORC ID: 0000-0001-9539-4345

ID $3^{\text {er }}$ Coautor: Hermes Orestes, Cedillo-Campos / ORC ID: 0000-0002-7547-6543

DOI: $10.35429 / J T I P .2020 .11 .4 .13 .20$

Recibido 30 de Agosto, 2020; Aceptado 20 Diciembre, 2020

\section{Resumen}

La falta de profesionales especializados en temas clave para la industria moderna con la entrada de la cadena de suministro digital y de acelerados cambios en la tecnología es una variable en la mayoría de los países latinoamericanos. En el ámbito internacional ha emergido el "sistema de aprendizaje dual" modelo que combina la formación teórica de la universidad con la práctica en la organización, de forma que se fortalece los conocimientos de los aprendices para asegurar los requisitos empresariales $\mathrm{y}$, a la vez, desarrollen su propia empleabilidad. El objetivo del artículo es dar a conocer la aceptación del modelo dual en el estado de San Luis Potosí cubriendo el programa educativo en Técnico Superior Universitario en Logística área Cadena de Suministros en base a entrevistas y encuestas aplicadas a los alumnos y al personal interno de la organización. Como resultado, se aplicó la metodología deductiva para la identificación de variables fundamentales con el fin de desarrollar un modelo analítico que busca determinar los procesos de la cadena logística dentro del ámbito organizacional.

Modelo dual, San Luis Potosí, Logística

\begin{abstract}
The lack of professionals specialized in key issues for modern industry at the entrance of the digital supply chain and of accelerated changes in technology is a variable in most Latin American countries. At the international level, the "dual learning system" model has emerged that combines the theoretical training of the university with the practice in the organization, in a way that strengthens the knowledge of the apprentices to ensure business requirements and, at the same time, develop their own employability. The objective of the article is to publicize the acceptance of the dual model in the state of San Luis Potosí, covering the educational program in Higher University Technician in Logistics in the Supply Chain area based on interviews and surveys applied to students and internal staff of the organization. As a result, the deductive methodology was applied for the identification of fundamental variables in order to develop an analytical model that seeks to determine the processes of the logistics chain within the organizational scope.
\end{abstract}

Dual model, San Luis Potosí, Logistics

Citación: CHAVÉZ-GOVEA, Roberto, CASTRO-LÓPEZ José David, SALAZAR-SALAS Elizabeth y CEDILLOCAMPOS, Hermes Orestes. Impacto del modelo dual de TSU en logística área cadena de suministros en la ciudad de San Luis Potosí. Revista de Tecnologías en Procesos Industriales. 2020. 4-11: 13-20

$\uparrow$ Investigador contribuyendo como primer autor. 


\section{Introducción}

Los efectos de la cuarta revolución industrial se perciben dentro de las organizaciones establecidas en México. Por un lado las tecnologías cognitivas, llámese automatización, robótica o inteligencia artificial, ha tomado mucha relevancia en organizaciones de todos los sectores. Las organizaciones enfrentan la imperiosa necesidad de evolucionar el conocimiento colectivo que quieren proveer a su fuerza laboral para comunicarla a candidatos potenciales, clientes y actores involucrados. Y por otro lado no se percibe un aumento tan fuerte como en otros países como es la fuerza laboral flexible como estrategia de competitividad.

Es importante destacar la importancia del inicio de la formación dual dentro de la concepción del programa educativo en Técnico Superior Universitario en Logística área Cadena de Suministros impartida en el estado de San Luis Potosí en el 2018, en el cual está basada en educación-trabajo, en las que establece un proyecto profesional en dicha modalidad de estudio y de preparación.

El comité de Transformación Curricular (2007) menciona, "hoy día el país requiere de nuevos modelos educativos pertinentes, viables $y$ de calidad que contribuyan a las políticas sociales, científicas, tecnológicas y culturales a nivel regional, nacional e internacional en un mundo globalizado" y esto involucra evaluar al sector empresarial.

De acuerdo al ranking mundial de talento elaborado por el IMD World Talent Ranking (2020), México se posicionó del lugar 60 al lugar 56 de 63 economías evaluadas, en donde se evalúa la capacidad para desarrollar y atraer talento. Para 2020, las economías más competitivas en conocimiento son las que se plantean en el desarrollo del talento en cada etapa del proceso educativo.

\section{MEXICO}

OVERALL PERFORMANCE (63 countries)

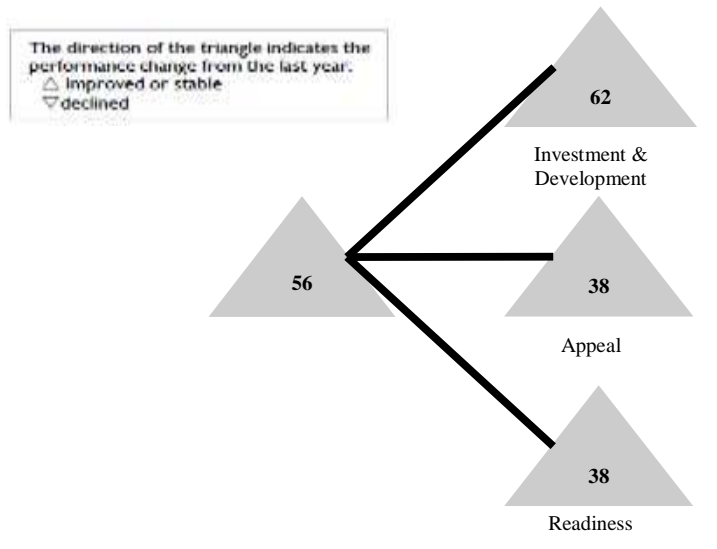

Figura 1 Overall Performance

Fuente: IMD 2020

La recapacitación se ha vuelto un imperativo para seguir creciendo y no quedar fuera de cambios importantes en cada sector de la economía. El talento con habilidades especializadas o complejas es difícil de encontrar en el mercado laboral mexicano, San Luis Potosí tuvo una tasa de desocupación muy baja del 3.0\% en octubre de 2019 (INEGI, 2019). Este fenómeno se presenta junto con una creciente demanda de trabajadores altamente calificados en el manejo de tecnologías (Bassi, Busso, Urzúa, y Vargas, 2012) e implica que el sistema educativo mexicano no provee a todos sus estudiantes los conocimientos y destrezas exigidos por el mercado laboral.

Por lo que, genera aún más preguntas sobre la teleología educativa, el principio que persiguen los seres humanos al participar en la educación. La respuesta por parte del gobierno estatal a esta pregunta ha sido propulsar la reformar a la ley de educación estatal para establecer la educación dual, ajustar los planes y programas de estudio a la vocación de la región, aprovechar mejor los convenios con las organizaciones y desarrollar un observatorio laboral para monitorear los perfiles de egreso, los programas educativos, ofrecer alternativas laborales a egresados, con especial énfasis en el primer empleo y en mejores condiciones, pero sobre todo evaluar a las instituciones a través de indicadores de empleabilidad (SEGE, 2016).

Un actor importante en estos cambios han sido las mismas empresas mexicanas y extranjeras que participan en la toma de decisiones educativas a través de diversos mecanismos directos e indirectos. 
Entre ellos, el sector empresarial se hace presente en asociaciones como el Consejo Coordinador Empresarial y la COPARMEX, o el banco Santander, que tiene presencia en México a través de la UNAM. De modo similar, la SEP estableció en 2014 un Consejo Nacional de Participación Social en la Educación (CONAPASE) en el cual participa el sector empresarial.

En la organización, los jóvenes aprendices conocen de manera automática la cooperación con otros especialistas, al resolver dificultades, el trato con distintos niveles jerárquicos y el desarrollo de alianzas. Saber tratar de manera hábil a gremios y superiores, además de otras habilidades, también forma parte del desarrollo de competencias sociales (Pfeiffer 2017).

\subsection{Sistemas de formación actuals}

El sistema de capacitación con base en competencias, modular y centrada en resultados (CBC - Capacitación Basada en Competencia) tiene espacio únicamente en el proceso de enseñanza-aprendizaje. Los alumnos obtienen competencias por dos vías: a través de la colaboración de diversos entornos, y a través de sus profesores, instructores y facilitadores. Continuando con una visión constructivista del aprendizaje, los alumnos deben adueñarse del conocimiento que proviene en primera instancia de sus profesores. El proceso de aprendizaje que debe de fomentarse en un programa $\mathrm{CBC}$, los modelos $\mathrm{y}$ las simulaciones pueden ser aditamentos valiosos para la estrategia.

El modelo de aprendizaje dual (o formación profesional dual) es un proceso para desarrollar aprendices en profesiones y oficios en los que participan de forma coordinada universidad y organismos industriales. El alumno, denominado "aprendiz", recibe la formación teórica $30 \%$ en la universidad y la práctica $70 \%$ en el lugar de trabajo. Las organizaciones ofertan espacios de trabajo a través de un convenio de formación a los aspirantes que desean convertirse en aprendices por lo que conlleva una doble tutoría. El tutor industrial lo desarrolla a las necesidades de la organización define objetivos, el tutor académico orienta para dar estructura a la formación de los propósitos fijados.
Una de las particularidades principales que diferencian a ambos sistemas (CBC y el modelo dual) es el método pedagógico, o proceso en que se transfieren las habilidades que se adquieren en los procesos de aprendizaje a las actividades que se llevan a cabo de manera diaria en el trabajo.

Los sistemas de formación dual muestran mejores resultados de aprendizaje y empleabilidad a largo plazo. Sin embargo, no es posible replicar uno a uno este sistema de un país a otro. Es por esto, que en América Latina se habla del "modelo dual" y no del "sistema dual" (Hans Lindemann, 2019).

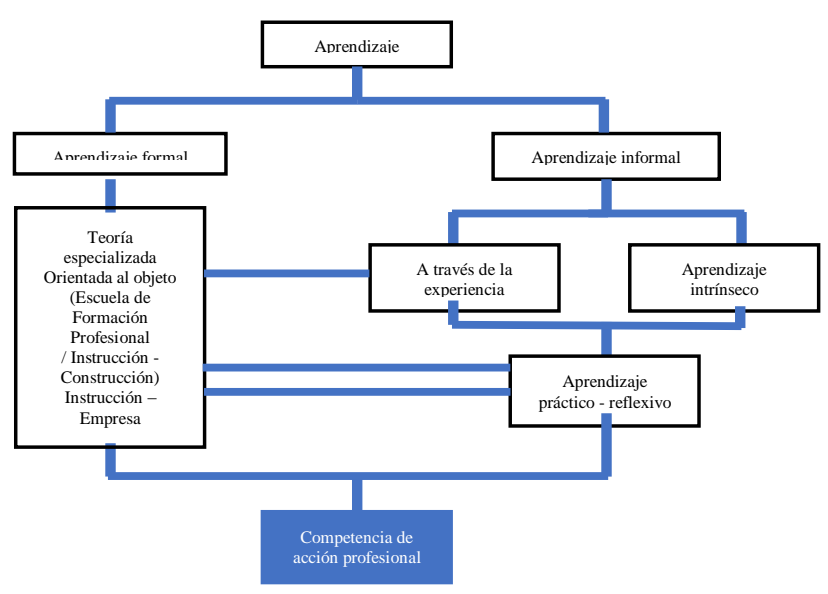

\section{Metodología}

Se realiza la investigación en el presente documento, exponiendo el método análisis deductivo considerando la importancia y las razones por las que es imprescindible el modelo dual en Logística área Cadena de Suministros, para fortalecer e incrementar el vínculo con las empresas del Estado de San Luis Potosí.

El artículo toma como referencia el análisis de requerimientos y expectativas del sector laboral de la zona de influencia de la Universidad Tecnológica de San Luis Potosí (UTSLP), con relación a la pertinencia del programa educativo de TSU en Logística área Cadena de Suministros. 
Documento trabajado en conjunto con el sector educativo representado por profesores de tiempo completo del área Logística y con el sector empresarial representado por 22 expertos del sector industrial de las ramas de Transporte, Tráfico, Almacén, Comercio Exterior, Planeación y Compras, los cuales, describieron $\mathrm{y}$ definieron de manera precisa las funciones, tareas y operaciones que el especialista en Logística debe ser capaz de realizar, y se fundamenta en la información obtenida del taller "Análisis Situacional del Trabajo (AST)", efectuado en las instalaciones de la Universidad Tecnológica de San Luis Potosí.

Es importante señalar que el Análisis Situacional del Trabajo (AST) es la herramienta clave implementada por el Subsistema de Universidad Tecnológicas y Politécnicas a través de su Dirección General de Universidades Tecnológicas y Politécnicas que permite conocer puntualmente las necesidades de los diversos sectores que demandan recurso humano calificado, en este caso específico el del sector logístico, con el objetivo de ampliar las posibilidades de incorporación y permanencia en el mercado laboral de los egresados de dicho sector.

Para el Subsistema es de vital importancia, que los planes de estudio contemplen los requerimientos y expectativas del sector productivo, por lo que la información que integra el reporte de AST es generada y validada por el personal representante de las organizaciones del sector productivo participante, un ejemplo de ello es que con el apoyo de técnicas de trabajo grupal como lo es la tormenta de ideas desarrollada con los expertos del sector empresarial, se identifican y validan de manera consensuada las funciones y tareas clave a realizar por los aprendice en el sector logístico, siendo algunos ejemplos las siguientes:

Administrar estrategias de mejora continua enfocadas a los sistemas de gestión.

- Gestionar los elementos que componen la cadena de valor para desarrollar la competitividad empresarial.

Gestionar los procesos de compras para garantizar el abastecimiento óptimo.

Controlar aspectos fundamentales del comercio internacional para su correcta aplicación y competitividad de las organizaciones.
- Planear el abastecimiento de productos en cada fase de la cadena.

Administrar el almacén para garantizar disponibilidad de producto, aumentando ventas, nivel de servicio y optimización de la operación.

- Implementar modelos de gestión de inventarios para garantizar el control de las existencias.

- Gestionar los requerimientos de empaque y embalaje para salvaguardar las condiciones físicas de los materiales.

- Gestionar los procesos de distribución, para optimizar el flujo de los materiales y de los costos.

- Gestionar el transporte en la cadena de suministros para optimizar los flujos de materiales.

- Administrar el personal para asegurar la excelencia operacional.

El análisis realizado, permite la continuidad de las acciones emprendidas para modificar el sistema educativo mexicano, ya que actualmente, no es posible para la educación superior permanecer al margen del desarrollo del sector productivo. Esto conlleva a determinar la necesidad de tener jóvenes con las competencias en el área de logística, necesarias para lograr un buen desempeño laboral.

Cabe señalar la importancia de la participación de las empresas para lograr la pertinencia de los planes y programas de estudio vigentes, lo cual es indicativo de la nueva mentalidad del sector educativo y productivo en la realización de tareas comunes como la formación de los futuros profesionales técnicos que se insertarán en sus empresas.

Lo anteriormente expuesto evidencia no solo la importancia de la pertinencia que debe tener el programa de estudios del Técnico Superior Universitario en Logística área Cadena de Suministros con el mercado laboral, si no también la necesidad de contar con programas de estudios que se encuentren fuertemente vinculados al sector productivo como lo es modelo de educación dual cuyo principal objetivo es brindar a los aprendices la oportunidad de poder practicar en escenarios reales los conocimientos adquiridos en las aulas de clase, considerando además que la logística es un sector muy dinámico y sobre todo indispensable para el mundo global actual. 
El modelo de educación dual es una herramienta clave para contribuir con la disminución de la problemática de escasez de talento que tanto preocupa al sector empresarial. En el año de 2018 se acreditó por parte de la DGUTyP el permiso a la Universidad Tecnológica de San Luis Potosí para vincular la carrera de TSU en Logística área Cadena de Suministros al modelo dual, logrando ser la primera universidad en el Estado en integrar estudiantes de Técnico Superior Universitario en Logística área Cadena de Suministros en las áreas logística de las organizaciones.

Con base en los modelos existentes, se diseña y desarrolla el plan de estudio basado en las necesidades detectadas, utilizando los conocimientos en áreas de estudio que determinan las materias que el aprendiz debe dominar, la universidad asigna docentes con experiencia laboral y expertos en las áreas y temas actuales que competen al sector logístico, por otra parte las actividades a realizar por el aprendiz, ( ejecutar, aprender a hacer), en el lugar de trabajo para las áreas logísticas, busca generar beneficio a la organización, como emplear personal con las competencias específicas, ahorro de costos de reclutamiento y capacitación,

Así como beneficiosa para el estudiante al desarrollar competencias específicas para el empleo, mayor vinculación con las empresas y la obtención de una constancia.

\subsection{Proceso interno de Modelo Dual en la UTSLP}

Téenicos Superios Universitarios (TSU):

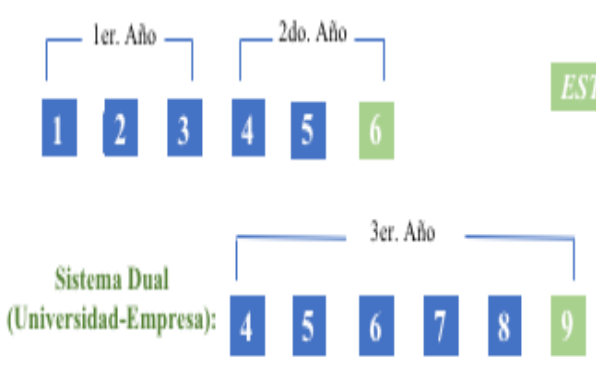

TSU

Figura 3 Extracto ¿Cómo se estudia el sistema dual? Fuente: UTSLP

\subsection{Proceso de tutoría en el Modelo Dual}

La función tutorial incluye temas complementarios de habilidades blandas como liderazgo, comunicación, motivación, integración y el trabajo en equipo, con el objetivo de cumplir la formación integral. La tutoría académica trabaja en vinculación con el instructor de la empresa para dar seguimiento a los aprendices.

Una vez en la empresa, los aprendices deben seguir un plan de formación de trabajo diseñado y preestablecido por la organización y tomar cursos-talleres que complementen el desarrollo de competencias específicas de las diferentes áreas del sector logístico que existen en la organización.

Existen varios cursos de competencias laborales que el personal de la planta les imparten a los aprendices y que llevan una secuencia, mismos que deberán aplicar durante su estancia en ella, con el objetivo de adquirir las nuevas competencias específicas que demanda la empresa.

En el transcurso del cuatrimestre el aprendiz es enviado a diferentes departamentos para conocer las funciones que se realizan y para aplicar lo aprendido en los cursos. Se visita tanto a la empresa como al alumno para dar seguimiento puntual al programa y se retroalimenta al asesor industrial sobre los resultados obtenidos por los aprendices.

Una vez terminado el proceso el aprendiz debe realizar su estadía profesional, con el objetivo de realizar un proyecto de mejora en alguna de las áreas logísticas de la empresa; aplicando todos los conocimientos adquiridos de sus cursos de competencia laboral y las prácticas realizadas.

Las mejoras realizadas son documentadas y señalando el impacto que genera el proyecto para la empresa. Una vez que el aprendiz ha terminado con todo el proceso, tanto en la universidad como en la organización, se hace acreedor de un título profesional de nivel TSU y una Constancia por parte de la empresa que certifica que cumplió con el programa del modelo dual. 


\section{Análisis del Modelo Dual}

Hoy en día las actividades logísticas en la cadena de suministro en la organizaciones, requieren operar productivamente, la integración del modelo dual asegura la aplicación de mejora continua, herramienta de control de calidad, en la que se monitorean las actividades, la ejecución y se verifican los resultados, para poder asegurar procesos controlados y detectar la oportunidad de mejora, llevando a cabo acciones preventivas y correctivas que lleven al cumplimiento de resultados para la satisfacción del cliente. Mejorar constantemente y siempre el sistema de producción y servicio, para mejorar la calidad y la productividad, y así reducir los costes continuamente. (Deming, 1986).

Se analizará la información del modelo dual por medio de encuestas realizadas a los actores participantes, en este caso, los instructores como eje fundamental que en colaboración con los aprendices enfatizan en áreas específicas de la cadena de suministros, donde el aprendiz-colaborador desarrolla proyectos para la mejora continua en las organizaciones, el instructor verifica y confirma por medio de la encuesta el cumplimiento de las necesidades y expectativas del sector empresarial, información en la que se basa la organización para tomar la decisión de continuar integrando estudiantes del modelo dual en Logística y Cadena de Suministros, la encuesta muestra información sobre el desempeño del modelo y del mismo aprendiz, realizando los siguientes planteamientos; $1 .-i$ En qué nivel considera que se cumplieron las expectativas que usted tenía sobre el proyecto planteado?, refiriéndose a usted como instructor en la organización, en esta la opción de respuesta se plantea como Excelente equivalente al $100 \%$, hasta regular con valor de $70 \%$ ó menos, el resultado del análisis de esta información se muestra en la siguiente gráfica, en la se tiene un total de 111 asesores industriales encuestados, de los cuales 59 indican que las expectativas se cumplen en nivel excelente, 44 encuestados indican que se cumplen en el nivel de muy bien y 8 asesores indican que se cumple bien únicamente, en conclusión el 93\% de los encuestados confirma que se cumplen la expectativas del modelo, calificando entre Excelente y Muy bien.
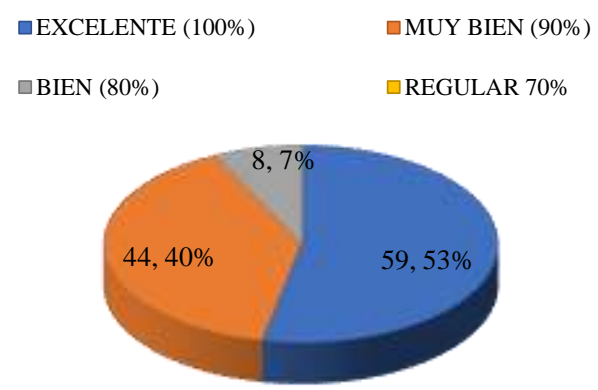

Gráfico 1 Expectativa Organizacional Fuente: Elaboración Propia

Con una integración de alrededor de 405 aprendices al sector productivo y continuando con el análisis de los datos que indica que el aprendiz-colaborador del modelo dual de la UTSLP, cumple con los conocimientos y habilidades detectadas por el sector empresarial y educativo, lo que se valida con las respuestas obtenidas en el planteamiento que indica; " $¿ \mathrm{La}$ organización volvería a solicitar aprendicescolaboradores para llevar a cabo el modelo dual incluyendo la especialidad en Logística área cadena de suministros?", información que se presenta en la siguiente gráfica, donde podemos visualizar que 109 de los 111 encuestados confirman que los estudiantes del modelo dual si tienen el nivel de conocimiento esperado.

घ Continuidad de Integración $\quad$ nivel de conocimiento

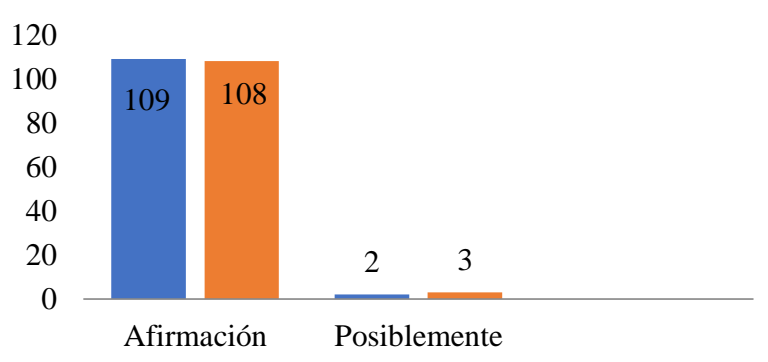

Gráfico 2 Encuesta a instructores industriales Fuente: Elaboración Propia

\section{Resultados}

El modelo dual Técnico Superior Universitario en Logística área Cadena de Suministros, tiene como finalidad dar el vínculo y la integración a las empresas del sector logístico y a los egresados de un modelo enfocado a la enseñanza durante la relación aprendiz-empresa, basado en las demás especialidades que la Universidad Tecnológica ha integrado a este modelo con éxito, año con año se integran más empresas que apoyan en la vinculación del modelo y así el número de estudiantes se mejora, se muestran los datos a continuación en la Gráfica 3. 
Egresados de Modelo Dual

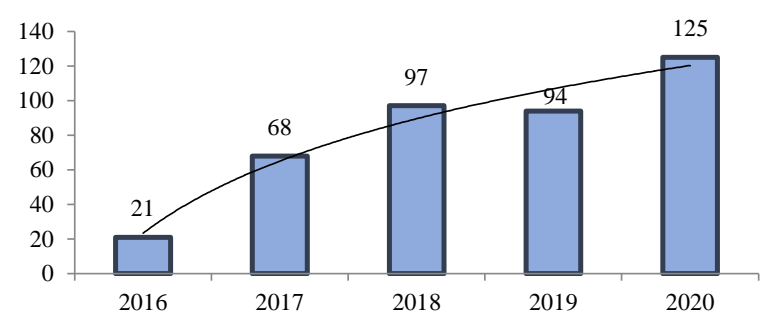

Gráfico 3 Egresados de Modelo Dual Fuente: Elaboración Propia

Gracias a la implementación del modelo dual en la Universidad Tecnológica de San Luis Potosí y al sector empresarial, se ha logrado incorporar alrededor de 405 estudiantes, 35 de ellos pertenecientes al TSU en Logística área Cadena de Suministros, ratificando que cumplen con los requerimientos de la empresa para el desarrollo de los proyectos y actividades, incluyendo todos los procesos logísticos, desde la logística de entrada: compras, desarrollo de proveedores, logística interna: planificación y control de la producción, control de inventarios y en la logística de salida: almacén, planeación de rutas, transporte, distribución, última milla etc.

El presente éxito del modelo, ha llevado a obtener resultados puntuales que conllevan beneficios para todos los actores involucrados en este proceso, cabe destacar proyectos de alto impacto como: Actualización de Kanban y Estandarización del proceso, que se ha realizado en una de las organizaciones del sector automotriz donde el cumplimiento del objetivo consistió en optimizar inventarios y espacios en el supermercado, para disminuir paros de líneas de producción, a través del desarrollo y la implementación de herramientas potencialmente aplicadas por el TSU en Logística área Cadena de Suministros, como diseño de lay out, definición y cálculo de stock mínimo-máximo, aplicadas por el aprendiz y guiadas por el asesor industrial, obteniendo como resultado el incremento de la capacidad en el supermercado de un 10.97\%, logrando llevar a cabo el abastecimiento a producción en tiempo y cantidad requerida al $100 \%$, y disminuir en automático los paros de línea, eliminando los costos por tiempos muertos y pérdidas monetarias generadas por estos, una vez estandarizado este proceso se monitorea para seguimiento y control.
Otro logro importante de nuestros aprendices en dicho sector, específicamente en el área de materiales, es el proyecto de implementación y estandarización de rutas de entrega, la necesidad de hacer eficiente el proceso lleva a los involucrados a definir como objetivo, el implementar la ruta del tugger para que el material se entregue y devuelva en el tiempo y espacio asignado en cada línea de producción, con el fin de reducir paros de línea, retrabajos, tiempos muertos, disminuir costos y a su vez cuidar la ergonomía del trabajador, el cual fue realizado en cuatro de veintidós líneas de producción, involucrando el almacén general, donde el aprendiz en conjunto con su instructor industrial realiza la propuesta de actividades claves de logística interna:

1. Implementación y estandarización de rutas para optimización de proceso y reducción del tiempo ciclo.

2. Establecer por medio de hoja de trabajo estándar, las actividades de cada trabajador involucrado en el proceso.

3. Reducir tiempo de ciclo, tiempos muertos y la cantidad de vehículos motorizados.

Estas actividades marcan el resultado final del proyecto, a través de DMAIC (Metodología seis sigma), basado en la secuencia metodológica, definir, medir, analizar, mejorar, obteniendo una reducción del $42.5 \%$, dato significativo en el tiempo ciclo de surtimiento de material, lo que lleva a la reducción del uso de un tugger menos, lo que representa ahorros importantes a la organización. El resultado, desde el inicio de la generación de Técnicos Superior Universitarios en el modelo dual de Logística área Cadena de Suministros ha culminado con al menos 15 proyectos que han impactado en las diferentes áreas Logísticas, como áreas de Kanbansupermercados, almacenes, inventarios, ruteos, compras, materiales, empaque y embalaje, etc.

\section{Conclusiones}

Actualmente se cuenta con 11 aprendices en el sector logístico, confirmando que la integración del modelo dual al programa educativo de TSU en Logística área Cadena de Suministros, cumple el propósito común de proveer egresados con competencias y habilidades específicas adecuadas para la empresa y así estimular a la implementación de mejoras y beneficios en las organizaciones de acuerdo a la siguiente ideología.

CHAVÉZ-GOVEA, Roberto, CASTRO-LÓPEZ José David, SALAZARSALAS Elizabeth y CEDILLO-CAMPOS, Hermes Orestes. Impacto del modelo dual de TSU en logística área cadena de suministros en la ciudad de San Luis Potosí. Revista de Tecnologías en Procesos Industriales. 2020 
"Estimular la educación y la automejora de todo el mundo". " Lo que necesita la organización no es solo gente buena; necesita gente que este mejorando su educación".( Deming, 1986).

Cabe señalar que la UTSLP, tiene una participación activa en el sector logístico, como prueba de ello pertenece a la Triple Hélice del Clúster Logístico de San Luis Potosí, por ello su gran compromiso por continuar generando egresados competitivos en el área logística y obtener mayor vinculación de empresas del sector y potencializar el Modelo Dual, el cual es apoyado por el Gobierno del Estado de San Luis Potosí, para que más estudiantes tengan la oportunidad de ser partícipes de este tan importante modelo de educación.

Con lo anteriormente expuesto, podemos concluir que con la inclusión del Modelo Dual al programa de TSU se contribuye a cerrar la brecha de las necesidades de talento y disminuir la escasez de personal capacitado en áreas del sector logístico, con lo que se proyecta que el Modelo Dual incorporado a la Universidad Tecnológica de San Luis Potosí en Logística área Cadena de Suministros cumple las expectativas esperadas de los tres actores; institución educativa, aprendiz y empresa.

\section{Referencias}

Bassi, M., Busso, M., Urzúa, S., \& Vargas, J. (2012). Desconectados: Habilidades, educación y empleo en América Latina. Washington: BID Educación.

Comité de Transformación Curricular. (2007). Propuesta de documento rector. Transformación curricular facultad de ciencias exactas y naturales. Medellín Consultado el 20 de noviembre del 2020. Disponible en http://huitoto.udea.edu.co/programacionacadem ica/contenido/IPPA/ACUERDOS

TRANSFORMACION

CURRICULAR/documento-maestro-rector/Doc Rector - Fac Ciencias Exactas.pdf

Dehnbostel, Peter, Betriebliche Bildungsarbeit. Kompetenzbasierte Aus- und Weiterbildung im Betrieb. Segunda Edición, Baltmannsweiler: Schneider, 2015.
Deloitte. (2019). Tendencias en Capital Humano México. Consultado el 20 de noviembre del $2020 . \quad$ Disponible en https://www2.deloitte.com/content/dam/Deloitt e/mx/Documents/human-capital/HCT_2019.pdf

Hans Lindemann. (2019). El modelo dual. ¿Una alternativa para la crisis de la formación profesional en México?. Consultado el 25 de noviembre del 2020. Disponible en http://library.fes.de/pdf-

files/bueros/mexiko/15586.pdf

IMD World Talent Ranking (2020). Scores countries across three factors of Investment \& Development, Readiness and Appeal. Consultado el 23 de noviembre del 2020. Disponible en https://www.imd.org/globalassets/wcc/docs/rele ase-

2020/talent/imd_world_talent_ranking_2020.pd $\mathrm{f}$

INEGI. (2019). Series desestacionalizadas de la tasa de desocupación total trimestral según entidad federativa. Consultado el 25 de noviembre del 2020. Disponible en https://www.inegi.org.mx/app/tabulados/default .html?nc=625

Pfeiffer, Sabine; Ritter, Tobias et.al, "Betrieb lernen”, Hans-Böckler-Stiftung Nr 366, 2017.

SEGE. (2016). Programa sectorial de educación. Consultado el 20 de noviembre del 2020. Disponible https://slp.gob.mx/SEGE/PDF/Programa Sectorial de Educación 2015-2021.pdf

William Edwards Deming,. Calidad, Productividad y Competitividad La salida de la crisis. (1989).Traducción Jesús Nicolau Medina Ediciones Díaz de Santos, S.A. 